\title{
Estratégias em saúde no combate e prevenção das violências contra as mulheres
}

\author{
Health strategies in combate and prevetions violences against women \\ Estrategias de salud en la combate y prevención de violencias contra las mujeres
}

Recebido: 20/01/2021 | Revisado: 30/01/2021 | Aceito: 03/02/2021 | Publicado: 10/02/2021

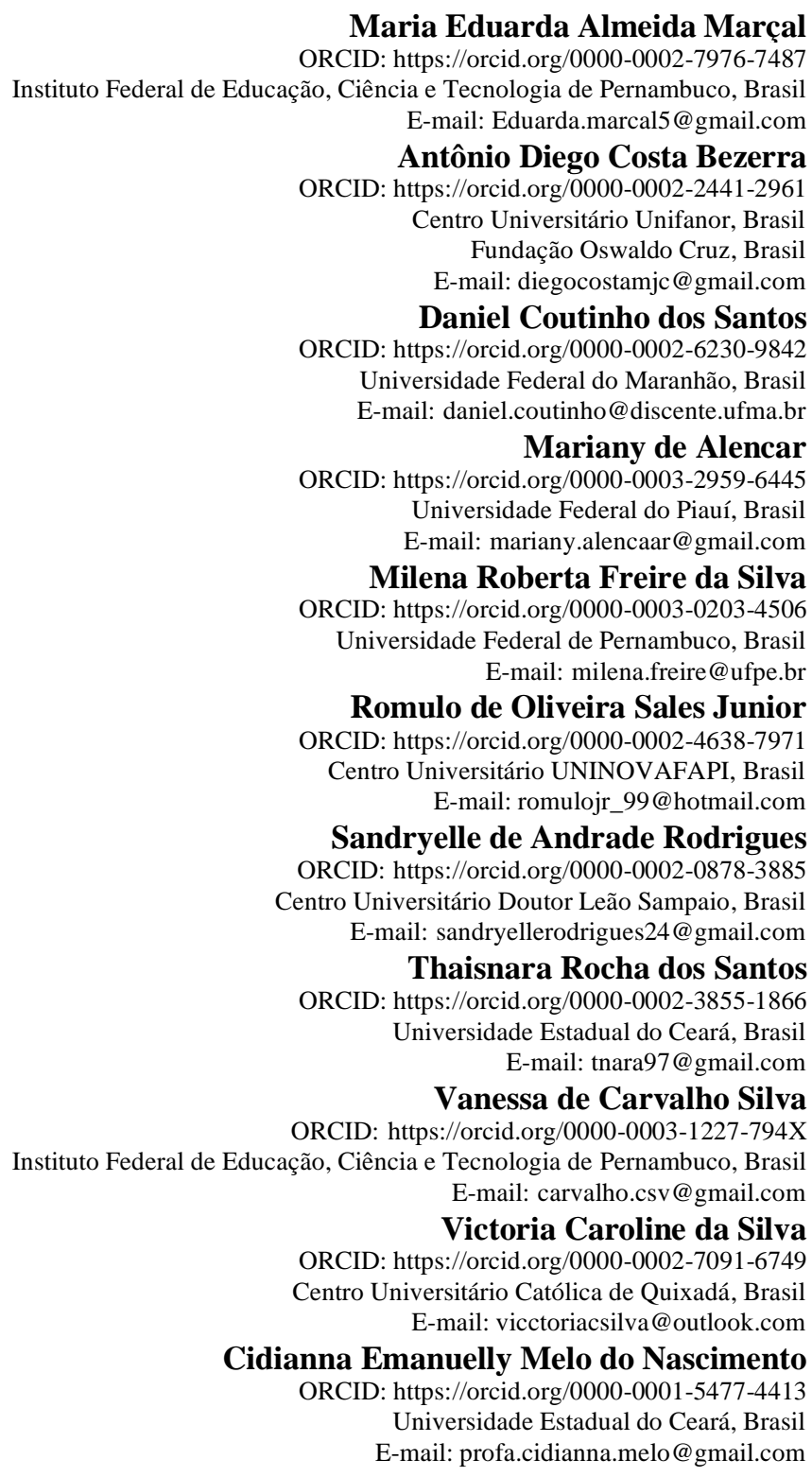

\begin{abstract}
Resumo
Objetivo: Identificar quais as estratégias que estão sendo utilizadas para a minimização e prevenção da violência contra a mulher. Metodologia: Trata-se de uma revisão integrativa no qual esse método tem função de agregar e sumariar resultados de pesquisas sobre um tema ou questão demarcada. A pesquisa foi conduzida nas bases de dados BVS, onde se inclui a LILACS e MEDLINE, SciELO, e BDTD. Como descritores foram utilizados "Tecnologia em saúde" e "Violência contra a mulher" indexados nos DeCS, usou-se o operador booleano AND, para formar a estratégia de busca. Resultados: Violência obstétrica é ainda pouco reconhecida enquanto um ato violento, sendo necessário abordar seus direitos durante a gestação, parto e pós-parto. Discussão: Diante dos resultados, foi possível observar que as tecnologias são ferramentas que facilitam a comunicação entre as mulheres e os profissionais de saúde, já que a violência contra a mulher é um fenômeno multifatorial que exige a criação de estratégias para o
\end{abstract}


enfrentamento e prevenção. Conclusão: Observou-se que o uso de tecnologias no combate e prevenção da violência contra a mulher tem sido de grande credibilidade, pois facilita bastante a identificação pelos profissionais dessas violências ocorridas, trazendo assim, um melhor conforto e segurança para essas mulheres vulneráveis que muitas vezes tem dificuldade de expor essas agressões.

Palavras-chave: Violência contra a mulher; Tecnologia em saúde; Educação em saúde.

\begin{abstract}
Identify which technologies are being used to minimize and prevent violence against women. Methodology: This is an integrative review in which this method has the function of aggregating and summarizing research results on a specific topic or issue. The research was conducted in the BVS databases, which includes LILACS and MEDLINE, SciELO, and BDTD. As descriptors were used "Health technology" and "Violence against women" indexed in the DeCS, the Boolean operator AND was used to form the search strategy. Results: Obstetric violence is still little recognized as a violent act, and it is necessary to address your rights during pregnancy, childbirth and post childbirth. Discussion: In view of the results, it was possible to observe that technologies are tools that facilitate communication between women and health professionals, since violence against women is a multifactorial phenomenon that requires the creation of strategies for coping and prevention. Conclusion: It was observed that the use of technologies in the fight and prevention of violence against women has been of great credibility, as it facilitates the identification by professionals of these violence that greatly facilitates, thus bringing a better comfort and security for these vulnerable women than many sometimes it is difficult to expose these aggressions.
\end{abstract}

Keywords: Violence against women; Health technology; Health education.

\title{
Resumen
}

Identificar qué tecnologías se están utilizando para minimizar y prevenir la violencia contra las mujeres. Metodología: Se trata de una revisión integradora en la que este método tiene la función de agregar y resumir los resultados de la investigación sobre un tema o tema específico. La investigación se realizó en las bases de datos de BVS, que incluyen LILACS y MEDLINE, SciELO y BDTD. Como se utilizaron los descriptores "Tecnología sanitaria" y "Violencia contra la mujer" indexados en el DeCS, se utilizó el operador booleano AND para formar la estrategia de búsqueda. Resultados: La violencia obstétrica aún es poco reconocida como un acto violento, y es necesario atender sus derechos durante el embarazo, el parto y el posparto. Discusión: A la vista de los resultados, se pudo observar que las tecnologías son herramientas que facilitan la comunicación entre las mujeres y los profesionales de la salud, ya que la violencia contra la mujer es un fenómeno multifactorial que requiere la creación de estrategias de afrontamiento y prevención. Conclusión: Se observó que el uso de tecnologías en la lucha y prevención de la violencia contra las mujeres ha sido de gran credibilidad, ya que facilita la identificación por parte de los profesionales de estas violencias lo que facilita enormemente, brindando así una mayor comodidad y seguridad a estas mujeres vulnerables que muchas. a veces es difícil exponer estas agresiones.

Palabras clave: La violencia contra las mujeres; Tecnología sanitaria; Educación para la salud.

\section{Introdução}

A violência contra a mulher é considerada um problema grave de saúde pública. Isso deve-se principalmente devido à estruturação de gênero no decorrer da história, onde o homem é considerado superior a mulher em qualquer âmbito, seja na família, local de trabalho e até mesmo no hospital no ato do parto. Dessa forma, a violência se torna algo natural e na maioria das vezes aceitável para a população e para a família da vítima (Heisler, Costa, Jahn \& Arboit, 2017).

Consequentemente, muitas mulheres têm dificuldade de denunciar ou comentar sobre a violência sofrida, devido à falta de apoio das pessoas ao seu redor. Nesse contexto, há relato que essas vítimas que sofrem violência têm maior probabilidade de procurar mais os serviços de saúde para um cuidado do que um serviço policial para a denúncia e dessa forma, o âmbito hospitalar se torna mais propícios para a ruptura da discrição sobre a violência cometida. Assim, é de suma importância que os profissionais multidisciplinares das unidades hospitalares, estejam aptos e capacitados para receber e identificar essas mulheres fragilizadas devido à ocorrência da violência, buscando prestar um melhor cuidado de confiança e um acolhimento a essa mulher (Magalhães, Ramos, Feitosa, Souza \& Almeida, 2020).

No que se diz a respeito as estratégias tecnológicas, estão cada vez mais presentes no dia a dia da sociedade, tornando-se uma ferramenta necessária para ser trabalhada no âmbito hospitalar, principalmente referindo-se à prevenção, 
combate e educação em saúde, com o intuito de promover um cuidado centrado, inovador e com uma melhor qualidade para profissional e paciente (Santos \& Souza, 2017).

Dessa forma, as tecnologias são uma grande aliada dos profissionais de saúde no momento da identificação da violência contra a mulher, pois se torna uma ferramenta importante que pode contribuir no diálogo entre o profissional e a vítima, criando também um vínculo entre eles e buscando uma facilidade na identificação das violências cometidas (Magalhães et al., 2020). Isto posto, o objetivo do estudo é identificar quais as tecnologias que estão sendo utilizadas para a minimização e prevenção da violência contra a mulher.

\section{Metodologia}

Trata-se de uma revisão integrativa qualitativa no qual esse método tem função de agregar e sumariar resultados de pesquisas sobre um tema ou questão demarcada, de maneira ordenada, auxiliando para a aquisição de um maior conhecimento do tema trabalhado (Mendes, Silveira \& Galvão, 2008). Este estudo foi desenvolvido em 5 etapas: $1^{\text {a }}$ fase: Definição da questão norteadora "Como as estratégias em saúde podem contribuir para a política de enfrentamento da violência contra a mulher?"; $2^{\mathrm{a}}$ fase: Coleta de dados, onde foi definido as bases de dados e realizado as buscas dos artigos; $3^{\mathrm{a}}$ fase: Seleção dos estudos e por meio dos critérios de inclusão e exclusão estabelecidos; $4^{\mathrm{a}}$ e $5^{\mathrm{a}}$ Fase: Análise e elegibilidade dos estudos e por meio da leitura dos artigos e discussão dos resultados encontrados (Ribeiro, Gonçalves, Borges, Loreto \& Amaral, 2019)

Para identificação dos artigos foi utilizado a estratégia PICo que representa um acrônimo, onde: P (paciente, população ou problema) é a violência contra a mulher; I (intervenção) as estratégias em saúde e Co é contexto, ou seja, o combate e prevenção (Araújo, 2020).

A pesquisa foi conduzida nas bases de dados Biblioteca Virtual em Saúde (BVS), onde se inclui a LILACS (Literatura Latino-americana e do Caribe em Ciências da Saúde) e MEDLINE (Medical Literature Analysis and Retrieval System Online), SciELO (Scientific Electronic Library Online) e BDTD (Biblioteca Digital Brasileira de Teses e Dissertações). Como descritores foram utilizados "Tecnologia em saúde" e "Violência contra a mulher" indexados nos Descritores em Ciências da Saúde (DeCS), usou-se o operador booleano AND, para formar a estratégia de busca. Os critérios de inclusão para refinamento são trabalhos publicados nos últimos 5 anos, e serem na língua portuguesa. Foram excluídos estudos não relacionados ao objetivo da pesquisa. A coleta foi realizada no mês de novembro de 2020.

Desta forma ao realizar os cruzamentos nas bases de dados com os descritores estabelecidos, foi encontrado na BVS 16 artigos, sendo 6 correspondente a LILACS e 3 a MEDLINE, já na SCIELO foram encontrados 16 artigos, e na BDTD 28 artigos, obtendo assim 58 artigos ao total. No processo de triagem para identificar os artigos completos, disponíveis em Português, Inglês e Espanhol, e recorte temporal a partir de 2015, foram retirados 15 artigos, pois diferiam destes critérios e referente aos duplicados foram excluídos 18 artigos, resultando em 25 artigos. No quesito da elegibilidade dos estudos após a triagem foram lidos por completo para que fosse possível a análise do título que assim foi excluídos 9 artigos por não corresponderem e, analisado o resumo onde foram excluídos mais 9 artigos, obtendo assim 7 artigos para serem incluídos na síntese deste estudo, pois são que abordam estratégias para o combate e prevenção da violência contra a mulher.

Os artigos incluídos foram classificados quanto ao nível de evidência através de um sistema de classificação que leva em consideração 7 níveis: nível I - evidências provenientes de revisões sistemáticas ou meta análises de ensaios clínicos randomizados relevantes; nível II - evidências obtidas de ensaios clínicos bem delineados; nível III - evidências originadas de ensaios clínicos bem delineados, porém sem randomização; nível IV - evidências derivadas de estudos do tipo caso controle e coorte bem delineados; nível V-evidências procedentes de revisões sistemáticas de estudos descritivos e qualitativos; nível VI 
- evidências retiradas de um único estudo qualitativo ou descritivo; e nível VII - evidências propostas pela opinião de autoridades e/ou relatórios emitidos por comitês de especialistas (Melnyk \& Fineout-overholt, 2005).

\section{Resultados e Discussão}

O Quadro 1 demonstra os artigos que foram encontrados que abordam estratégias tecnológicas para o combate e prevenção da violência contra a mulher.

Quadro 1: Descrição de autores, ano de publicação, título, link de acesso para o artigo, conclusão e o nível de evidência.

\begin{tabular}{|c|c|c|c|}
\hline Autor/ Ano & Título / Link de acesso & Conclusão & Nível de evidência \\
\hline $\begin{array}{l}\text { SANTOS, A. L.M.; } \\
\text { \& SOUZA, M. H.T. } \\
\text { DE; / } 2017\end{array}$ & $\begin{array}{l}\text { Elaboração de novas tecnologias } \\
\text { em enfermagem: utilização de } \\
\text { uma cartilha para } \\
\text { prevenção/https://pesquisa.bvsalu } \\
\text { d.org/portal/resource/pt/biblio- } \\
1031893\end{array}$ & $\begin{array}{l}\text { Violência obstétrica é ainda } \\
\text { pouco reconhecida enquanto um } \\
\text { ato violento, sendo necessário } \\
\text { abordar seus direitos durante a } \\
\text { gestação, parto e pós-parto, Dai } \\
\text { a importância dessa Cartilha } \\
\text { Educativa }\end{array}$ & VI \\
\hline $\begin{array}{l}\text { HEISLER, E. D.; } \\
\text { et. al. / } 2017\end{array}$ & $\begin{array}{l}\text { Potencialidades e limites da visita } \\
\text { domiciliar para identificar e } \\
\text { abordar mulheres em situação de } \\
\text { violência/ } \\
\text { https://pesquisa.bvsalud.org/portal } \\
\text { /resource/pt/biblio-967660 }\end{array}$ & $\begin{array}{l}\text { A inclusão da visita domiciliar } \\
\text { na ESF como tecnologia leve- } \\
\text { dura, voltada às vítimas, } \\
\text { possibilita aprimorá-la como } \\
\text { técnica de abordagem do } \\
\text { problema. Neste sentido, podem } \\
\text { subsidiar os profissionais para } \\
\text { qualificar esta prática. }\end{array}$ & $\mathrm{V}$ \\
\hline $\begin{array}{l}\text { MAGALHÃES, } \\
\text { V.M. P. R.; et al. / } \\
2020\end{array}$ & $\begin{array}{l}\text { Validação de álbum seriado para } \\
\text { enfermeiros da atenção básica } \\
\text { sobre violência doméstica contra } \\
\text { a mulher/ } \\
\text { https://pesquisa.bvsalud.org/portal } \\
\text { /resource/pt/biblio-1124594 }\end{array}$ & $\begin{array}{l}\text { O álbum seriado intitulado } \\
\text { "Violência Doméstica contra a } \\
\text { Mulher" foi considerado } \\
\text { adequado para subsidiar os } \\
\text { enfermeiros nas estratégias de } \\
\text { educação e promoção da saúde. }\end{array}$ & $\mathrm{V}$ \\
\hline SENA, L. M. / 2016 & $\begin{array}{l}\text { "Ameaçada e sem voz, como num } \\
\text { campo de concentração": a } \\
\text { medicalização do parto como } \\
\text { porta e palco para a violência } \\
\text { obstétrica. } \\
\text { https://bdtd.ibict.br/vufind/Record } \\
\text { /UFSC_a3c0738ef821b15ed9b37 } \\
\text { b43013b8787 }\end{array}$ & $\begin{array}{l}\text { Uma estratégia simples, que não } \\
\text { há grandes modificações } \\
\text { estruturais e é sugerida é a } \\
\text { capacitação de estudantes e } \\
\text { profissionais para que atuem na } \\
\text { elaboração conjunta, com as } \\
\text { gestantes, de seus planos de } \\
\text { parto. }\end{array}$ & $\mathrm{V}$ \\
\hline
\end{tabular}




\begin{tabular}{|c|c|c|c|}
\hline $\begin{array}{l}\text { MANFRINI, D. B. / } \\
2017\end{array}$ & $\begin{array}{l}\text { 'Somos todxs Adelir': partir de si e } \\
\text { ação política frente à violência } \\
\text { obstétrica em Florianópolis. / } \\
\text { https://bdtd.ibict.br/vufind/Record } \\
\text { /UFSC_e3c7873a8acb186c88a83 } \\
\text { e9297d5f5e7 }\end{array}$ & $\begin{array}{l}\text { O seguimento de discussões em } \\
\text { torno da violência obstétrica, } \\
\text { acesso à informação e respeito } \\
\text { às escolhas, com emfase na } \\
\text { autonomia das mulheres sobre } \\
\text { seus corpos e sustento para as } \\
\text { decisões que necessitam tomar } \\
\text { perante este, tanto da rede } \\
\text { familiar, social, institucional e } \\
\text { normativa. }\end{array}$ & VI \\
\hline $\begin{array}{l}\text { BRILHANTE, A. } \\
\text { V. M. / } 2015\end{array}$ & $\begin{array}{l}\text { Gênero, sexualidade e forró : um } \\
\text { estudo histórico social no } \\
\text { contexto nordestino. } \\
\text { https://bdtd.ibict.br/vufind/Record } \\
\text { /UFOR_84bb736f09b14951af20d } \\
\text { 879b213c0b3 }\end{array}$ & $\begin{array}{l}\text { Foi utilizado músicas de artistas } \\
\text { da classe musical do forró, por } \\
\text { meio de seleção específica. Com } \\
\text { isso é de suma importância para } \\
\text { a compreensão das } \\
\text { vulnerabilidades às quais as } \\
\text { mulheres estão expostas, buscar } \\
\text { as narrativas mestras e os } \\
\text { discursos hegemônicos. }\end{array}$ & VI \\
\hline $\begin{array}{l}\text { SANTOS, I. B. / } \\
2017\end{array}$ & $\begin{array}{l}\text { Violência Contra a Mulher ao } \\
\text { Longo da Vida: Estudo Entre } \\
\text { Usuárias da Atenção Primária. / } \\
\text { https://bdtd.ibict.br/vufind/Record } \\
\text { /UFES_04b310de01942faf108043 } \\
\text { 7cbe4a0b3e }\end{array}$ & $\begin{array}{l}\text { O estudo revelou altas } \\
\text { prevalências de violência por } \\
\text { parceiro íntimo, ao longo da } \\
\text { vida, entre as usuárias das } \\
\text { unidades de saúde da atenção } \\
\text { básica, é importante ponderar } \\
\text { que os profissionais de saúde } \\
\text { precisam } \\
\text { Compreender a violência contra } \\
\text { a mulher como objeto de } \\
\text { cuidado em saúde, que vai } \\
\text { muito além dos cuidados } \\
\text { referentes às consequências da } \\
\text { violência. }\end{array}$ & VI \\
\hline
\end{tabular}

Fonte: Autores.

Diante disto, foi possível observar que as tecnologias são ferramentas que facilitam a comunicação entre as mulheres e os profissionais de saúde, já que a violência contra a mulher é um fenômeno multifatorial que exige a criação de estratégias para o enfrentamento e prevenção. Nesse aspecto, os estudos realizados por Santos (2017) e Magalhães et al. (2020) utilizam álbuns seriados com ilustrações e diagramações sobre a violência contra mulher baseado na literatura científica e validado por um conjunto de especialistas, porém os autores realizaram as suas pesquisas em públicos-alvo diferentes. O público-alvo do primeiro estudo são as vítimas, o álbum seriado é utilizado com o intuito de investigação e identificação de altos índices de violência com os parceiros íntimos das vítimas.

Em relação da importância do profissional na temática, a pesquisa de Magalhães et al. (2020), faz a validação de um álbum seriado sobre violência doméstica contra a mulher voltado diretamente para o profissional enfermeiro utilizar durante suas consultas, visto que principalmente na atenção básica, encontra-se um contato maior entre o enfermeiro e a mulher que sofre violência, dessa forma, o álbum trata-se de uma tecnologia que irá facilitar a comunicação entre profissional e paciente, visando sempre a educação em saúde e prevenção da violência contra essas mulheres. 
No seguimento da atenção básica como no último estudo citado, a pesquisa de Heisler, et al. (2017), fez uso de tecnologia leves e leves-duras, que correspondem ao uso de saberes e conhecimento técnico, por meio de entrevistas aos profissionais de saúde que fazem parte das unidades básicas de saúde do município alvo do estudo, onde é questionado na realização das visitas domiciliares, é possível realizar a identificação de agressores e vítimas de violência, com isso é enfatizando a importância da escuta ativa e do vínculo entre profissional e usuário, para que a identificação seja com mais facilidade e de forma ágil.

As pesquisas realizadas por Manfrini (2017), Sena (2017), Santos e Sousa (2017), utilizam-se também métodos através da tecnologia educacional, com uma abordagem em cartilhas educacionais com temas na violência obstétrica. A cartilha educacional tem grande pertinência no quesito campanhas, pois auxilia as pessoas a obterem informações de diversos âmbitos, onde mesmo os indivíduos com baixa escolaridade conseguem compreender o assunto abordado. Essa abordagem se faz de uma estratégia simples, com materiais elaborados com embasamento na literatura científica.

Sena (2016) a partir de um site criado pela pesquisadora, realizou o convite do público do seu estudo da sua tese, no qual ela realizou entrevistas e coletou dados referentes a experiências ocorridas desde os atendimentos de pré-natal até o momento do parto, além do período de pós-parto das participantes. No resultado do estudo, foi identificado a ocorrência da violência obstétrica em um grupo de mulheres e assim a pesquisadora, realizou um momento de conscientização da violência, para as participantes conseguirem identificar as situações para poder denunciar.

Nessa perspectiva de Sena (2016), observa-se a grande relevância da utilização de tecnologias como os sites e a internet para a identificação dessas violências contra as mulheres, visto que, muitas mulheres ainda têm dificuldades de expor a violência sofrida para um profissional, seja ele de saúde ou de justiça, assim, fica mais cômodo para essas mulheres apresentarem a agressão sofrida de modo virtual, com uma maior confiança diminuindo assim, as suas inseguranças e medos.

As músicas se exploradas com o objetivo de análise há a identificação de mensagens que podem ser positivas, como também negativas, no estudo de Brilhante (2015) é reconhecido várias músicas brasileiras que descrevem a violência de gênero, principalmente no contexto nordestino, por ser reconhecido como um local rude. Portanto, é possível refletir e também identificar outras músicas que são semelhantes e demonstram e instigam a violência da mesma forma.

Por fim, por se tratar de tecnologias voltadas para o combate e a prevenção da violência contra a mulher, os estudos sobre a temática são escassos, devido especialmente a falta de preparo dos profissionais e os tabus da sociedade sobre essa questão, dessa forma, é necessário a elucidação de evidências que possam contribuir para o manejo do combate a prevenção dessas violências, visto que é um acontecimento bastante presente no contexto do nosso país.

\section{Conclusão}

Com base nos resultados, observou-se que o uso de tecnologias no combate e prevenção da violência contra a mulher tem sido de grande credibilidade, pois facilita bastante a identificação pelos profissionais dessas violências ocorridas, trazendo assim, um melhor conforto e segurança para essas mulheres vulneráveis que muitas vezes tem dificuldade de expor essas agressões.

Todavia, também foi observado a escassez de estudos que retratam essas tecnologias, principalmente voltado para o profissional de saúde, visto que este é a chave principal para o combate e a prevenção dessas violências, dessa forma, é necessário o aperfeiçoamento da temática pelos profissionais e pela ciência, para que essas novas formas de tecnologias sejam disseminadas e desenvolvidas, usufruindo de todas as ferramentas disponíveis, com o intuito de uma prestação de um melhor cuidado para essas mulheres que buscam ajuda. 
Isto posto, é necessário que haja o uso frequente destas estratégias tecnológicas, pois irão contribuir para o minimização e prevenção da violência contra a mulher, principalmente por parte dos profissionais de saúde que podem e devem ajudar a mulher a identificar a violência incentivando assim que ela faça a denúncia e haja o combate da violência.

\section{Referências}

Araújo, W. C. O. (2020). Recuperação da informação em saúde: construção, modelos e estratégias. Convergências em Ciência da Informação, 3 (2). https://doi.org/10.33467/conci.v3i2.13447.

Brilhante, A. V. M. (2015). Gênero, sexualidade e forró: um estudo histórico social no contexto nordestino (Tese de doutorado). Universidade de Fortaleza, Ceará, Fortaleza, Brasil. https://bdtd.ibict.br/vufind/Record/UFOR_84bb736f09b14951af20d879b213c0b3.

Heisler, E. D., Silva, E. B., Costa, M. C., Jahn, A. C. \& Arboit, J. (2017). Potencialidades e limites da visita domiciliar para identificar e abordar mulheres em situação de violência. Ciência, Cuidado e Saúde, 16 (3), 10.4025/cienccuidsaude.v16i3.35348.

Magalhães, V. M. P. R., Santos, R. F., Ramos, C. V., Feitosa, L. G. G. C., Lago, E. C., Souza, E. K. S. \& Almeida, C. A. P. L. (2020). Validação de álbum seriado para enfermeiros da atenção básica sobre violência doméstica contra a mulher. Cogitare Enfermagem, 25. 10.5380/ce.v25i0.62729.

Manfrini, D. B. (2017). 'Somos todxs Adelir': partir de si e ação política frente à violência obstétrica em Florianópolis (Tese de doutorado). Universidade Federal de Santa Catarina, Florianópolis, Santa Catarina, Brasil. https://bdtd.ibict.br/vufind/Record/UFSC_e3c7873a8acb186c88a83e9297d5f5e7.

Melnyk, B.M. \& Fineout-overholt, E. (2005). Making the case for evidence-based practice. Evidence-based practice in nursing \& healthcare. A guide to best practice. (2a ed.). Philadelphia: Lippincot Williams \& Wilkins.

Mendes, K. S., Silveira, R. C. C. P. \& Galvão, C. M. (2008). Revisão integrativa: método de pesquisa para a incorporação de evidências na saúde e na enfermagem. Texto \& Contexto - Enfermagem, 17(4). 10.1590/S0104-07072008000400018.

Ribeiro, K.R.A., Gonçalves, F.A.F., Borges, M.M., Loreto, R. G. O., Amaral, M.S. (2019). Pós-Operatório de Revascularização do Miocárdio: Possíveis Diagnósticos e Intervenções de Enfermagem. Revista Cuidado é Fundamental Online,11(3). http:// dx.doi.org/10.9789/2175-5361.2019.v11i3.801-808

Santos, A. L. M. \& Souza, M. H. T. (2017). Elaboração de novas tecnologias em enfermagem: utilização de uma cartilha para prevenção. Revista de Enfermagem - UFPE, .11(10). 10.5205/reuol.12834-30982-1-SM.1110201725.

Santos, I. B. (2017). Violência Contra a Mulher ao Longo da Vida: Estudo Entre Usuárias da Atenção Primária (Dissertação de mestrado). Universidade Federal do Espírito Santo, Vitória, Espirito Santo, Brasil. https://bdtd.ibict.br/vufind/Record/UFES_04b310de01942faf1080437cbe4a0b3e.

Sena, L. M. (2016) "Ameaçada e sem voz, como num campo de concentração": a medicalização do parto como porta e palco para a violência obstétrica (Tese de doutorado). Universidade Federal de Santa Catarina, Florianópolis, Santa Catarina, Brasil. https://bdtd.ibict.br/vufind/Record/UFSC_a3c0738ef821b15ed9b37b43013b8787. 